\title{
ARTICLE \\ Comparative Analysis of Scheduling Algorithms Performance in a Long Term Evolution Network
}

\author{
Bamidele Moses Kuboye* \\ Department of Information Technology, School of Computing, The Federal University of Technology, Akure, Nigeria
}

\section{ARTICLE INFO}

Article history

Received: 13 August 2021

Accepted: 14 September 2021

Published Online: 29 September 2021

Keywords:

Algorithms

LTE

Scheduling

Network

Performance

\section{Introduction}

The rapid growth of wireless broadband mobile communication is astronomical, as a result, Long Term Evolution (LTE) has been embraced by a lot of subscribers all over the world. The 3rd Generation Partnership Project (3GPP) developed LTE as an emerging wireless technology in the path of mobile broadband evolution. Long Term Evolution (LTE) was developed as an all IP network to achieve a higher data rate, low latency, scalable bandwidth and mobility as well as wide coverage ${ }^{[1]}$. The LTE network enhanced the data rate in other to provide the radio resources for various highly demanded services in other to give a level of satisfaction of Quality-of-Service (QoS) to all active subscribers. LTE uses Orthogonal Frequency Division Multiple Access (OFDMA), Single Carrier- Frequency Division Multiple Access (SC-FDMA)

\section{ABSTRACT}

The advancement in cellular communications has enhanced the special attention given to the study of resource allocation schemes. This study is to enhance communications to attain efficiency and thereby offers fairness to all users in the face of congestion experienced anytime a new product is rolled out. The comparative analysis was done on the performance of Enhanced Proportional Fair, Qos-Aware Proportional Fair and Logarithmic rule scheduling algorithms in Long Term Evolution in this work. These algorithms were simulated using LTE system toolbox in MATLAB and their performances were compared using Throughput, Packet delay and Packet Loss Ratio. The results showed Qos-Aware Proportional Fair has a better performance in all the metrics used for the evaluation.

in the Downlink (DL) and Uplink (UL) respectively ${ }^{[2]}$ LTE supports up to $300 \mathrm{Mbps}, 75 \mathrm{Mbps}$ in the downlink and uplink for data transmission respectively using a bandwidth from $1.25 \mathrm{MHz}$ to $20 \mathrm{MHz}$. These requirements meet the needs of diverse network operators with different bandwidth allocations to give support for different services to their subscribers ${ }^{[3,4]}$.

Most of the services on LTE involve high-speed data, multimedia services and so on. It is the last effort to the provision of 4 th generation $(4 \mathrm{G})$ radio network. The revolution towards $4 \mathrm{G}$ started with the UMTS $3 \mathrm{G}$ technologies increasing their data rates and improving network architecture to $3.5 \mathrm{G}$ with High Speed Packet Access (HSPA) and HSPA evolution. These networks moved into $4 \mathrm{G}$ LTE to attain data rates of $100 \mathrm{Mbps}$, $50 \mathrm{Mbps}$ in DL and UL respectively ${ }^{[5,6]}$. LTE used the Radio Resource Management (RRM) to manage the

*Corresponding Author:

Bamidele Moses Kuboye,

Department of Information Technology, School of Computing, The Federal University of Technology, Akure, Nigeria;

Email: bmkuboye@futa.edu.ng 
limited radio resources. Thus, improves the data rate and secure quality of service (QoS) provisioning. LTE downlink scheduling is a component of RRM responsible for the allocation of shared radio resources among their user equipment's (UEs) ${ }^{[4]}$. Furthermore, scheduling strategy plays a vital role in system performances such as throughput, delay, fairness, loss rate and so on. This paper described the features of LTE network that have direct impact on scheduling strategy in section 2. Section 3 described the related works on scheduling algorithms. Section 4 and 5 discussed the features QoS Aware Proportional Fair (QAPF), Exponential Proportional Fairness (EX/PF) and LOG rule and the performance metrics used for the simulation. In section 6 , performance of QAPF, EX/PF and LOG rule scheduling algorithms were analyzed and compared.

\section{LTE Architecture}

The architecture of LTE is founded on flat, Internet Protocol (IP) requirements of 3 GPP Technologies ${ }^{[2]}$. LTE system architecture is made up of Evolved UMTS Terrestrial Radio Access Network (E-UTRAN) and the Evolved Packet Core (EPC) as depicted in Figure $1^{[7,8]}$.

\subsection{Evolved Universal Terrestrial Radio Access Network (E-UTRAN)}

The E-UTRAN controls the radio communications between the mobile and EPC ${ }^{[9]}$. The E-UTRAN is the access network has evolved-NodeBs (eNodeBs) and User Equipments (UEs). Also, it supports orthogonal frequency-division multiple access (OFDMA), Multiple Inputs and Multiple outputs (MIMO), management of the radio resources as well as security of transmitted data ${ }^{[3]}$. It is the base station that manages radio resources as used in GSM and has connection to the UEs where network air interface roles is performed ${ }^{[3,11,12]}$. As seen in Figure 1, LTE-Uu is the radio link between the UEs and eNodeB.

\subsection{The Evolved Packet Core (EPC)}

The EPC is the core network and enables exchange of data packets with the internet as well as UE while maintaining a given QoS ${ }^{[3]}$. EPC contains Home Subscriber Service (HSS), Policy Control and Charging Rules Function (PCRF), Mobility Management Entity (MME), P-GW and Serving Gateway (S-GW) ${ }^{[12]}$. The Home Subscriber Server (HSS) is the central database that contains information on the network operator's subscribers while Packet Data Network (PDN) Gateway (P-GW). Access point name (APN) identifies each data packet and the serving gateway (S-GW) forwards data between enodeB and the P-GW. The S1 interface is used to connect the eNodeB to EPC as seen in Figure 1. The MME controls signaling messages and HSS. Some of the other functions of EPC are Network access control, authentication, authorization, admission control, policy and charging enforcement, packet routing and transfer,

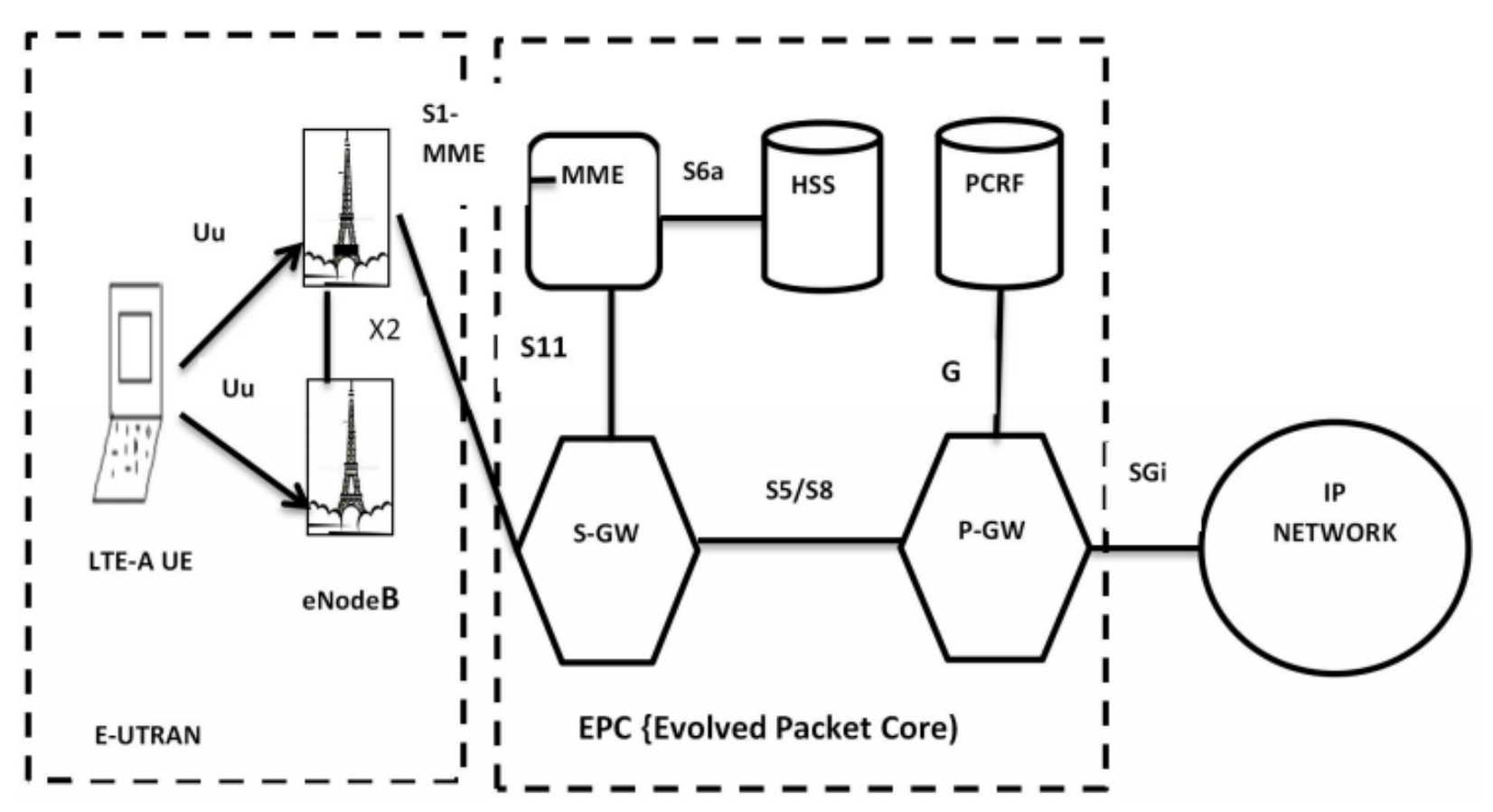

Figure 1. LTE Architecture ${ }^{[7]}$. 
security and others ${ }^{[8]}$.

\section{Related Works}

The deployment of an appropriate scheduling algorithm will make wireless communications more effective. Also, determination of the appropriate algorithm that provides the optimal use in the face of scarcity of radio resources will be of great value. Some previous work done by authors on scheduling algorithms that have shown appreciable improvements in literature are discussed in this section. A proportional fair (PF) scheduling algorithm built on INS was proposed by Wang et al ${ }^{[13]}$. In the proposed scheme, the fairness was improved without passing high difficulty to the system. The results of the simulation showed that the proposed algorithm can efficiently increase the throughput of LTE users as well as improve the system fairness. QoS aware proportional fair (QAPF) downlink scheduler for LTE network was by Myo and Mon ${ }^{[14]}$. Its purpose was to optimize the use of available resources while maintaining QoS requirements of different classes of service in GBR and non-GBR. The algorithm when compared with Modified- Largest Weighted Delay First (MLWDF) and Exponential (EXP) rule shows that a lower packet loss rate can be maintained for nonGBR bearers. At the same time, GBR bearers will have a high performance in delay and packet loss rate. Sadiq et al ${ }^{[15]}$ propounded a LOG rule algorithm, though similar to exponential (EXP) rule but uses logarithmic function on delay to calculate the scheduling parameters. It gave fairness to users when is in poor channel quality of service.

A new scheduling algorithm for downlink transmission in LTE was offered by Bechira et al ${ }^{[6]}$. This scheduler performance was evaluated and compared to Round Robin (RR), the opportunist Max rate and the Proportional Fair (PF) scheduler. The proposed algorithm improves the throughput in LTE system according to the simulation results. Elhadad et al ${ }^{[16]}$ proposed Enhanced Proportional Fair (E-PF) Scheduling Algorithm for LTE in order to enhance the capacity of LTE and the proposed scheduler was compared with the original Proportional Fair. The results showed improvement in the capacity of the LTE and as well as fairness to the distribution of the resources among the users. Sudheep and Rebekka ${ }^{[17]}$ presented a Proportional equal throughput (PET) scheduler using fair scheduling approach in LTE. This work modified Blind Equal Throughput (BET) algorithm and Proportional Equal Throughput (PET) algorithm emanated. The simulation showed that PET gives better fairness performances compared to BET without a significant decrease in system throughput.
An enhanced PF scheduling algorithm for LTE networks was proposed by de Oliveira et al ${ }^{[18]}$. The paper used the Latency-Rate (LR) server theory and system characteristics specified by the LTE standard for both theoretical and simulation investigations. The results show a better performance when compared with PF and MLWDF scheduler. Uyan and Gungor ${ }^{[19]}$ examined performances of some algorithms using throughput and fairness and thereafter, proposed a new QoS-aware downlink scheduling algorithm (QuAS). The simulation shows an increase in the QoS-fairness and overall throughput of the edge users without producing a substantial degradation in the system throughput when compared with best CQI, PF, RR and Coordinated Multi Point (CoMP) structure with RR. Yaqoob et al ${ }^{[20]}$ presented an enhanced EXPRULE (eEXPRULE) scheduling algorithm for real-time (RT) traffic in LTE network. The scheduler shows improvement on all the metrics used by most scheduler reviewed.

\section{Scheduling Algorithms Used}

Scheduling algorithm is not defined in LTE and various approaches have been presented to address this issue of scheduling algorithms ${ }^{[18,20]}$. Some results have shown some significant improvements in literature. Therefore, QAPF, EX/PF and LOG Rule are discussed for the purpose of this work:

\subsection{QoS Aware Proportional Fair (QAPF)}

QAPF is a downlink scheduler for LTE network proposed by Myo and Mon ${ }^{[14]}$. QAPF defines four MACQoS-traffic types as Voice over IP (VoIP), live video streaming, video streaming and e-mail as seen in Table 1. Firstly, QAPF differentiates different QoS classes by defining MAC bearer types as Guaranteed Bit Rate (GBR) and non-Guaranteed Bit Rate (nonGBR). GBR has Voice over Internet Protocol (VOIP), Live-video Streaming while nonGBR has video streaming and Email.

The QAPF directs the incoming IP packets into MAC QoS classes as shown Figure 2. Thereafter, the priority candidate lists are generated for the GBR and nonGBR bearer types in time domain (TD) scheduling. The TDS prioritized GBR and nonGBR using their Head of Line (HOL) delay. In GBR, the emergency bearers which have delayed closing to the maximum delay are first extracted.These extracted emergency bearers are sorted in descending order according to their delay. The priority for non GBR Pi, $t$, bearer $i$ at time $t$, is:

$$
P_{i}(t)=\operatorname{argMax}\left[w_{i} * \frac{r_{i}}{\overline{r_{i}}}\right]
$$


where, wi equals weight factor of nonGBR bearer i, $r i$ equals instant throughput and is the average throughput for bearer $i$. The time average throughput of user is updated by the moving average as:

$$
\overline{r_{i}}(t) \overline{=}(1-a) \bar{r}_{i}(t-a)+a r_{i}(t)
$$

where $a=\frac{2}{1+N}$ is scaling factor of $N$ time period.

The frequency domain (FD) assigned physical resource blocks to each user according to the priority list. The motive is to guaranteeing the QoS requirements of different service classes while maintaining the fairness and maximizing the system throughput.

Table 1. CQI to MAC-QoS Class Mapping

\begin{tabular}{ccccc}
\hline $\begin{array}{c}\text { Bearer } \\
\text { Type }\end{array}$ & Traffic Type & Priority & $\begin{array}{c}\text { Packet Delay } \\
\text { Budget }\end{array}$ & $\begin{array}{c}\text { Mac-QoS } \\
\text { Class }\end{array}$ \\
\hline \multirow{2}{*}{ GBR } & VOIP & 2 & $100 \mathrm{~ms}$ & Class 1 \\
& $\begin{array}{c}\text { Live-video } \\
\text { Streaming }\end{array}$ & 4 & $150 \mathrm{~ms}$ & Class 2 \\
& $\begin{array}{c}\text { Video Streaming } \\
\text { NonGBR }\end{array}$ & 7 & $300 \mathrm{~ms}$ & Class 3 \\
& Email & 8 & $300 \mathrm{~ms}$ & Class 4 \\
\hline
\end{tabular}

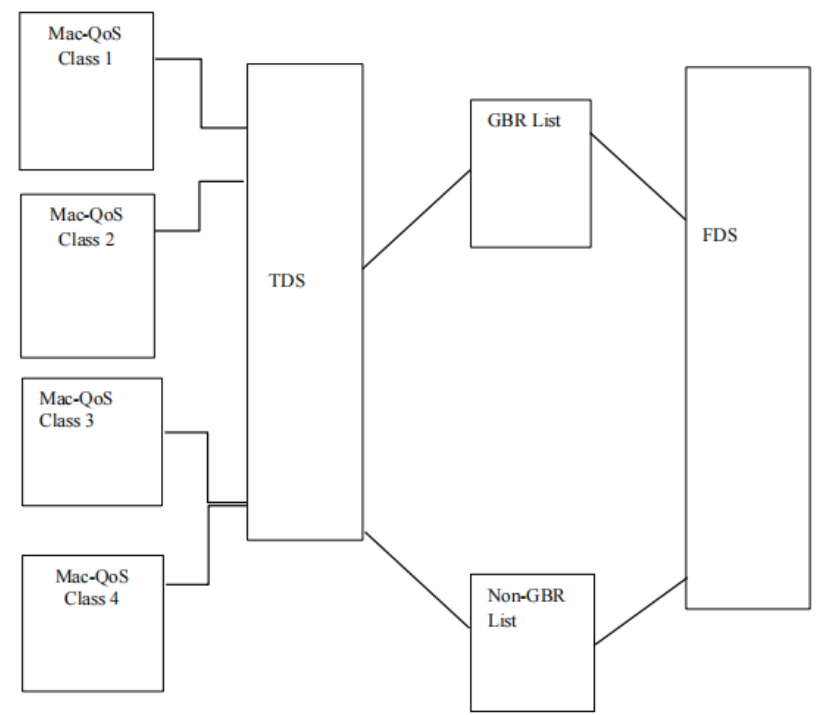

Figure 2. QAPF Mac-classes Framework ${ }^{[10]}$

\subsection{Exponential Proportional Fairness (EX/PF) Scheduler}

The EX/PF scheduling algorithm was proposed to give support to multiple traffic types so that Real-Time (RT) traffic will be prioritized over non-RT traffic ${ }^{[21]}$. Its metrics is calculated as follows:

$W_{i, j}=\exp \left(\frac{\alpha_{i} \mathrm{D}_{\mathrm{HoLi}}-X}{1+\sqrt{X} 9}\right) \frac{r_{i}(t)}{\overline{R_{i}}(t)}$

where $\mathrm{X}$ is given as:

$X=\frac{1}{N_{r t}} \sum_{i=1}^{N_{r t}} \alpha_{i} \mathrm{D}_{\mathrm{HoLi}}$
$\mathrm{N}_{\mathrm{rt}}$ denotes the number of active UEs for RT traffics, $\overline{R_{i}}(t)$ is the weight factor, is the previous average throughput of the user until time $t$ while stands for the expected data-rate for the user $\mathrm{i}$ at time $\mathrm{t}, \mathrm{D}_{\mathrm{HoLi}}$ expresses the head of line (HOL) packet delay, that is, difference between the current time and arrival time of a packet ${ }^{[22,23]}$.

\subsection{Logarithm Rule Scheduling Algorithm}

LOG rule algorithm balances QoS like delay and robustness. The algorithm allocates resources to users as EXP rule does with a prior knowledge of arrival and statistics of traffic channel ${ }^{[16]}$ :

$\mathrm{LOG}_{\text {rule }}=\max \left[b_{i} \log \left(c+\propto_{i} D_{H o L, i}\right) \frac{r(t)}{\overline{R_{i}}(t)}\right]$

where $\propto_{i}, b_{i}$ and $c$ are tunable parameters. Optimal parameters defined as

$\mathrm{b}_{\mathrm{i}}=\frac{1}{\frac{\mathrm{r}(\mathrm{t})}{\overline{\mathrm{i}_{\mathrm{i}}}(\mathrm{t})}}$ and $\mathrm{c}=1.1, \propto_{i}=\frac{5}{0.99 r_{i}}{ }^{[22,23]}$.

\section{Performance Metrics}

The following parameters where used:

Throughput (th) measures the rate of useful bits successfully transmitted through a network by a user per unit time. It uses Equation 6 for this:

Throughput $=\frac{B}{T \text { sim }}$

where B is the total amount of bits received while Tsim false is the total simulation time.

Average packet delay experienced by UE is the arrival time between the packets in the Queue to their departure. The average delay of the ith flow can be expressed by using Equation (7) ${ }^{[7]}$ :

$D_{i}=\frac{1}{N} \sum_{j=0}^{N}\left[T_{d}(j) \mid-T_{s}(j)\right]$

where $T_{s}(j) s$ stands for the time when the $\mathrm{j}$ 'th packet was transmitted from its source and $\mathrm{N}$ is the number of packets used.

Packet Loss Ratio (p) indicates the percentage of packets that missed their deadlines and is calculated as:

$P=\left(\frac{p_{\text {transmit }}-P_{\text {receive }}}{p_{\text {transmit }}}\right) \times 100$

where $p_{\text {transmit }}$ false is total size of packets transmitted, while $P_{\text {receive }}$ false is the total size of packets arrived ${ }^{[22]}$.

\section{Results of the Simulation}

In this section, the performance of QAPF, LOG-RULE and enhanced proportional fair (EX-PF) are compared. The LTE system toolbox in MATLAB is used using the 
parameters stated in Table 2. The LTE system Toolbox is an ideal application to simulate, analyze, and test the physical layer of LTE networks. It is also suitable to accelerate LTE algorithm and verify designs, prototypes, implementations compliance with the LTE standard ${ }^{[24]}$.

Table 2. Simulation parameters

\begin{tabular}{cc}
\hline Parameters & Value \\
\hline Bandwidths & $20 \mathrm{MH}_{\mathrm{z}}$ \\
Operating Frequency & $2 \mathrm{GH}_{\mathrm{z}}$ \\
Numbers of RB & 25 \\
Scheduling Time(TTI) & $1 \mathrm{~ms}$ \\
Number of Slot Carrier & 300 \\
Slot Duration & $1 \mathrm{~ms}$ \\
\hline
\end{tabular}

The first analysis evaluated the throughput performance of QAPF, EX-PF and LOG Rule. The simulation results are displayed in Figure 3. It is observed that the throughputs for the three algorithms are quite close. However the QAPF performed better than the EX-PF and the LOG rule.

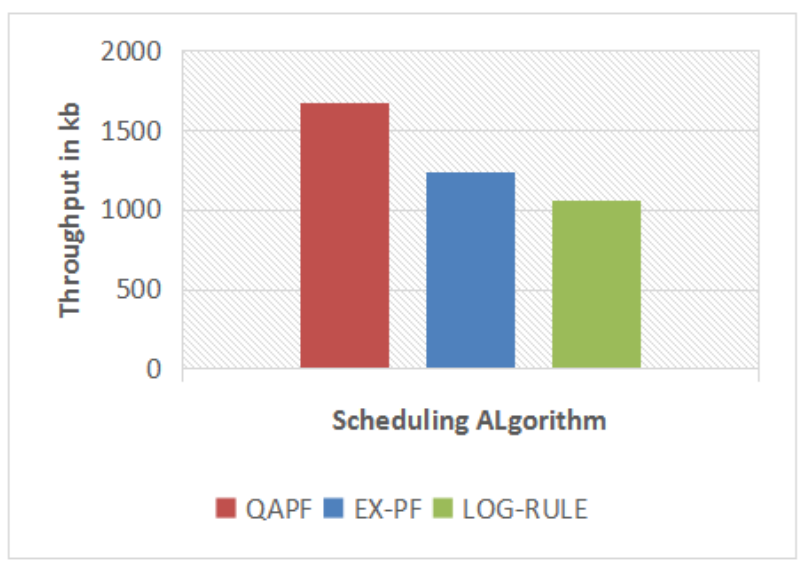

Figure 3. Throughput

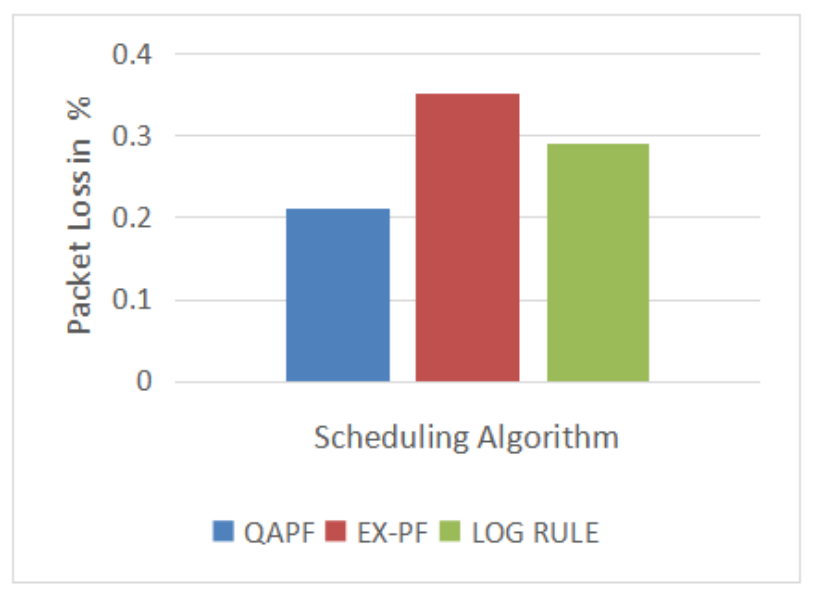

Figure 4. Packet Loss ratio

The second analysis evaluated the Packet Loss Ratio of QAPF, EX-PF and LOG rule shown in Figure 4. The lower the PLR value, the better the scheduler, EX-PF has a highest Packet loss ratio followed by LOG rule and QAPF. Therefore, QAPF performed better than EX-PF and $\mathrm{LOG}$ rule.

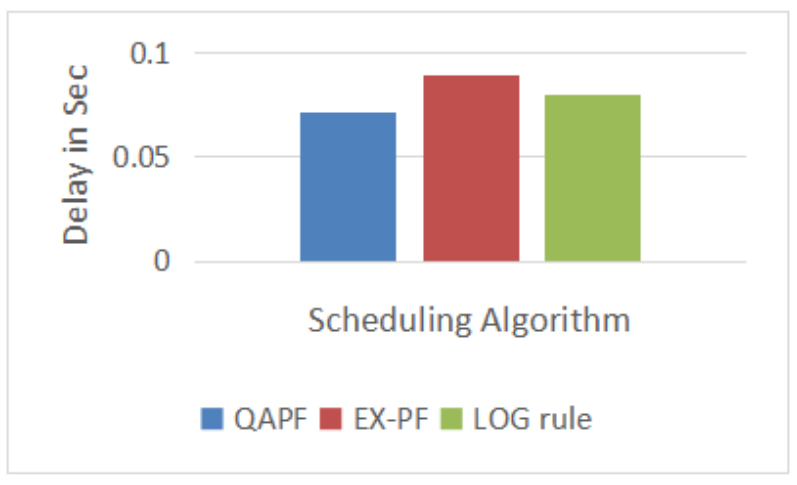

Figure 5. Delay Algorithm

Third analysis evaluated the packet average delay and it was observed that EX-PF has the highest Delay followed by LOG rule and QAPF. QAPF performs better than EX$\mathrm{PF}$ and LOG Rule as shown in Figure 5.

\section{Conclusions}

The performances of the algorithms were evaluated and compared using packets loss, average delay and throughput. The following discoveries were made: QAPF has the highest throughput, lowest average delay and PLR when compared to EX-PF and LOG Rule. EX-PF has the highest PLR and delay while LOG Rule has the least performances in all the three metrics. In a reliable communication such as data and voice, throughput and packet delivery are very important in which QAPF is more appropriate according to the results of the research.

\section{References}

[1] Lawal M.A, Saidu I. Mohammed A., and Sade Y.A.(2017). Downlink Scheduling Algorithms in LTE Networks: A Survey IOSR Journal of Mobile Computing \& Application (IOSR-JMCA) e-ISSN: 23940050, P-ISSN: 2394-0042. Volume 4, Issue 3 PP 0112 www.iosrjournals.org.

[2] Sulthana S. F. and Nakkeeran R. (2014). Study of Downlink Scheduling Algorithms in LTE Networks, Journal Of Networks, Vol. 9, No. 12.

[3] Mousavi H., Iraj S. Amiri, M.A. Mostafavi , C.Y. Choon (2019). LTE physical layer: Performance analysis and evaluation Applied Computing and Informatics 15 pp 34-44.

[4] Chadchan S. M. and Akki C. B. (2013). A Fair 
Downlink Scheduling Algorithm for 3GPP LTE Networks, I.J. Computer Network and Information Security, 2013, 6, 34-41. Published Online May 2013 in MECS (http://www.mecs-press.org/).

DOI: 10.5815/ijcnis.2013.06.05.

[5] Kuboye, B. M. (2019). Long Term Evolution (LTE) Network Evaluation in the South-West Region of Nigeria, European Journal of Engineering Research and Science, 4(3), 86-92.

DOI: https://doi.org/10.24018/ejers.2019.4.3.1160.

[6] Bechira N., Nasreddinea M., Mahmouda A., Walida H. and Sofiena M. (2014) Novel Scheduling Algorithm for 3GPP Downlink LTE Cellular Network Procedia Computer Science 40 ( 2014 ) 116-122.

[7] Kuboye, B.M. (2018) Performance Evaluation of Scheduling Algorithms for 4G (LTE). Communications and Network, 10, 152-163. https://doi. org/10.4236/cn.2018.104013.

[8] Zyren J. (2007). "Overview of the 3GPP Long Term Evolution Physical Layer" White Paper, fre csale semiconductor.

[9] Matin M. A. (2014). Chapter 1 Evolution of Wireless and Mobile Communications", IGI Global.

[10] Mousavi H., Amiri I. S., Mostafavi M.A., Choon C.Y. "2019". LTE physical layer: Performance analysis and evaluation, Applied Computing and Informatics.

[11] Zhou (2012). From LTE to LTE-A, LTE-Advanced Air Interface Technology.

[12] Aggarwal C. and Srivastava K.(2016). Securing IOT devices using SDN and edge computing, International onference on Next Generation Computing Technologies (NGCT).

[13] Wang H. Z., Sun Z. H., Wang J., and Liu, F. (2014). Study on the Resource Scheduling Algorithm for LTE-Advanced Downlink Systems. Applied Mechanics and Materials, 577, 1017-1021. https://doi. org/10.4028/www.scientific.net/amm.577.1017.

[14] Myo I. M. and Mon M. T (2015). Qos-Aware Proportional Fair (QAPF) Downlink Scheduling Algorithm For Lte Network Proceedings Of Seventh The IIER International Conference, Singapore, 3rd January 2015, ISBN: 978-93-84209-80-3.

[15] Sadiq B., Madan R., and Sampath A.., (2009). Downlink Scheduling for Multiclass Traffic in LTE, Eurasip Journal of Wireless Communication Networks, vol. 2, pp. 9-13, Oct. 2009.
[16] Elhadad M. I., El-Rabaie E.M., Abd-Elnaby M. (2014). Enhanced PF Scheduling Algorithm for LTE Downlink System, Mobile Computing Vol. 3.1, www.mc-journal.org.

[17] Sudheep S. and Rebekka B., (2014) "Proportional equal throughput scheduler - A very fair scheduling approach in LTE downlink," International Conference on Information Communication and Embedded Systems (ICICES2014), 2014, pp. 1-6.

DOI: 10.1109/ICICES.2014.7034111.

[18] de Oliveira R. P., de Góis L. A. and Foronda A. (2018). Enhanced PF Scheduling Algorithm for LTE Networks, International Journal of Communication Networks and Information Security (IJCNIS) Vol. 10, No. 1.

[19] Uyan O.G., Gungor V.C. QoS-aware LTE-A downlink scheduling algorithm: A case study on edge users. Int $\mathrm{J}$ Commun Syst. 2019. https://doi.org/10.1002/dac.4066.

[20] Yaqoob J. I. A.Y., Pang W. L., Wong S. K. and K. Y. Chan (2020) Enhanced exponential rule scheduling algorithm for real-time traffic in LTE network, International Journal of Electrical and Computer Engineering (IJECE) Vol. 10, No. 2, pp. 1993 2002 ISSN: 2088-8708.

DOI: 10.11591/ijece.v10i2.pp1993-2002.

[21] Kuboye, B.M., A. J. Gabriel, A. F. Thompson, and V. O. Joseph (2018). Analysis of Algorithms in Long Term Evolution (LTE), Journal of Computer Science and Its Application, 25(2), 59-71, ISSN: 20065523. https://www.ajol.info/index.php/jcsia/article/ view/179867.

[22] Mohd A, Mohd H O, Muhammed I. A, Rahmat B., (2014). Performance comparison of Downlink Packet Scheduling Algorithms in LTE Network, Proceeding of International Conference on Electrical Engineering, Computer Science and Informatics (EECSI 2014), Yogyakarta, Indonesia.

[23] Nwawelu U. N., Ani C. I. and Ahaneku M. A., (2017). Comparative Analysis of the Performance of Resource Allocation Algorithms in Long Term Evolution, Nigerian Journal of Technology (NIJOTECH), Vol. 36, No. 1, pp. 163-171. http://dx.doi. org/10.4314/njt.v36i1.21.

[24] MathWorks. Simulink Documentation. Available at https://www.mathworks.com/help/lte/ accessed on 9/9/2021. 